\title{
Interaction between luminance gratings and disparity gratings
}

\author{
MICHAEL WRIGHT $^{1, *}$ and TIM LEDGEWAY ${ }^{2}$ \\ ${ }^{1}$ Department of Human Sciences, Brunel University, Uxbridge, Middlesex UB8 3PH, UK \\ ${ }^{2}$ School of Psychology, University of Nottingham, University Park, Nottingham NG7 2RD, UK
}

Received 27 July 2002; revised 27 October 2002; accepted 28 October 2002

\begin{abstract}
It was shown from geometry and photographic measurement that the shading pattern for a sinusoidal corrugated surface of frequency $f$ approximates to a luminance-defined grating of frequency $f, 2 f$ or $f+2 f$ in specific relative phase. It was confirmed that a luminance grating modifies the appearance of a suprathreshold stereoscopic corrugated surface, suggesting an interaction between shading and binocular disparity. Disparity thresholds for detecting random-dot, disparitydefined gratings of spatial frequency 0.2 or $0.4 \mathrm{c} / \mathrm{deg}$ were measured in the presence of luminance gratings of spatial frequency $0.4 \mathrm{c} / \mathrm{deg}$ with the same orientation. Phase-specific facilitation of disparity thresholds was greatest for a phase relationship inconsistent with shading of a corrugated surface, and was disrupted by positional uncertainty. The presence of texture-defined lines (which served to mark explicitly the successive spatial locations of salient depth features in the image) produced a similar pattern of facilitation, in the absence of shape-from-shading cues. The pattern of results indicates direct local interactions, including spatial cueing, rather than interaction of depth cues.
\end{abstract}

Keywords: Shading; stereopsis; luminance; disparity; grating; surface.

\section{INTRODUCTION}

One possible strategy to explore the way the visual system combines different kinds of information is to study interactions between the most elementary stimuli in each class. Random-dot stereograms were originally devised in order to isolate stereoscopic depth cues from monocular cues to shape (Julesz, 1971). The introduction of sinusoidal, luminance-defined gratings in vision research likewise led to the isolation and analysis of early vision mechanisms sensitive to spatial contrast (Robson, 1966; Campbell and Robson, 1968). In this paper we consider whether a luminance grating added to a disparity-defined grating alters disparity-

\footnotetext{
${ }^{*}$ To whom correspondence should be addressed.
} 
detection thresholds. We consider three possible ways that such an interaction might occur. First, a visible contrast pattern may affect the detection of a spatially corrugated disparity pattern by spatial cueing effects (Burgess and Ghandeharian, 1984; Pelli, 1985). Second, there may be a direct spatial interaction between luminance contrast and disparity, such that disparity thresholds are affected by the simultaneous presence of a luminance pattern (Rohaly and Wilson, 1999; Kelly and Grossberg, 2000). Thirdly, appropriate shading may facilitate the detection of the disparity grating by contributing to a common representation of depth.

We will show that a stimulus composed of a sinusoidal luminance grating added to a disparity grating is a good approximation to the shading and disparity cues generated by an actual sinusoidal corrugated surface. Visual systems need to recover the three-dimensional structure of the environment from depth cues such as binocular disparity (stereopsis), motion parallax, texture and shading. An important question is how these various depth cues interact. For example, there is a close relationship between stereopsis and motion parallax (Rogers and Graham, 1979, 1982; Graham and Rogers, 1982; Bradshaw and Rogers, 1996). Evidence suggests that motion parallax and stereopsis are strongly inter-linked at an early stage of processing. Interactions between binocular disparity, perspective and texture gradient cues have also been studied (Cumming et al., 1993; Johnston et al., 1993). This work suggests that texture gradients and perspective can combine with disparity information under some circumstances.

Interactions between shading and binocular disparity have been reported by a number of researchers. Bülthoff and Mallot $(1988,1990)$ studied the interaction between shading and disparity depth cues using shaded ellipsoids. They concluded that both shading and disparate shading can generate depth, but edge-based disparities can override local shading as a depth cue. Shading cues are known to interact with, or augment, stereoscopic information when both are consistent (Todd et al., 1997; Doorschot et al., 2001). One difference between disparity and shading information is that there is no direct correspondence between shading and relative depth. Thus integration of shading and disparity information may affect representations of curvature or shape rather than depth (Tittle et al., 1998).

The aim of the present experiments was to determine whether a luminance grating can influence (a) the detection threshold and (b) the suprathreshold appearance of disparity-defined gratings, and whether this interaction is phase specific. Control conditions are included to distinguish local interactions and spatial cueing from cue-combination effects.

\section{MODELLING AND OBSERVATION OF IMAGE LUMINANCE FOR CORRUGATED SURFACES}

Consider a Lambertian corrugated surface of uniform reflectance, and spatial frequency $f$ that is horizontal, perpendicular to the line of sight and illuminated by a single directional light source. Because the surface luminance at each point in the 
image is proportional to the cosine of the angle of incidence (Marr, 1982; p.244), there will be a periodic spatial variation in luminance in the direction orthogonal to the orientation of the corrugations. The predominant spatial frequency of the luminance waveform will be either $f$ or $2 f$, depending on the direction of the light source relative to the surface. The illumination pattern for such a surface may be calculated from basic trigonometry. Let the surface be $A \sin \theta$. Then, the slope of the surface is $A \cos \theta$. The angle of the slope to the horizontal is atan $(A \cos \theta)$, and the angle between an incident light ray $\alpha$ and the surface (perpendicular $=0)$ is $\operatorname{atan}((A \cos \theta)-\alpha)$. So the illumination at the surface is proportional to $\cos (\operatorname{atan}((\mathrm{A} \cos \theta)-\alpha))$ (Fig. 1). Negative values of this expression occur where the light source is below the surface horizon and consequently the surface is in shadow. Some interesting results follow. For a corrugation frequency of $f$, the predominant frequencies in the luminance waveform are at $f$ and $2 f$. The first harmonic predominates in the illumination pattern when the direction of the light source is oblique (e.g. $45 \mathrm{deg}$ ) relative to the plane of the surface. That is, a luminance component with a frequency $f$ will predominate because the sides of the corrugations will be unequally illuminated. The peaks of the $f$ luminance component always correspond with one or other of the zero-crossings of the surface depth waveform. The phase of the $f$ component shifts by $\pi$ radians as $\alpha$ changes sign (i.e. direction through the perpendicular). When the illumination is perpendicular to the surface $(\alpha=0)$ the second harmonic predominates. The peaks of this $2 f$ luminance component always correspond with the peaks and troughs of the depth waveform. These relationships are depicted in Fig. 1a.

A different situation obtains under conditions of diffuse lighting (Langer and Bülthoff, 2000). Assume a uniform diffuse lighting source, such as the stereotypical grey British sky. The amount of light reaching a point on a surface depends on the amount of the sky visible from that point on the surface. Construction of the 'limit rays', that is, the tangents to the surface visible from a given point along the surface (see Fig. 1b), leads to a solution for the luminance profile, following a 'darker is deeper' rule (Tyler, 1998; Langer and Bülthoff, 2000). The balance between diffuse and directional lighting can shift the peak of the luminance waveform away from the peak of the surface (diffuse) and towards the zero-crossing of the surface (directional). A third factor that contributes to the illumination pattern is mutual reflection from the illuminated surface and this reduces the contrast of the shading pattern. There may also be a specular component to the surface reflectance function. There are good models to account for all these factors (Phong, 1975; Neumann and Neumann, 1995). Rather than attempt complete modelling, we have observed and photographed a uniform reflectance matt cardboard sinusoidal surface in different orientations under an actual grey British sky (Fig. 2a, b) using a Nikon Coolpix 995 digital camera under auto settings. The observed pixel greyscale values were measured on one-dimensional profiles of the resulting .jpg image files using ImageJ software, available at http://rsb.info.nih.gov/ij/. These profiles confirm our theoretical analysis with a predominance of $2 f$ and $f$ frequencies in specific phase 


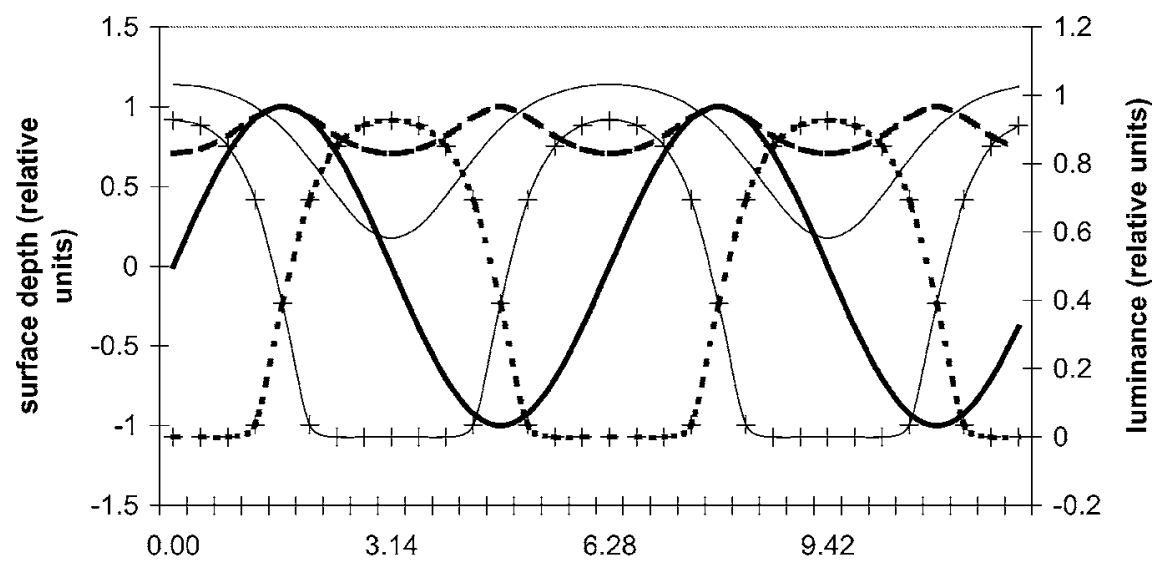

radians

- surface - - $0 \mathrm{deg}-22.5 \mathrm{deg}+67 \mathrm{deg}=+\cdot-67 \mathrm{deg}$

(a)

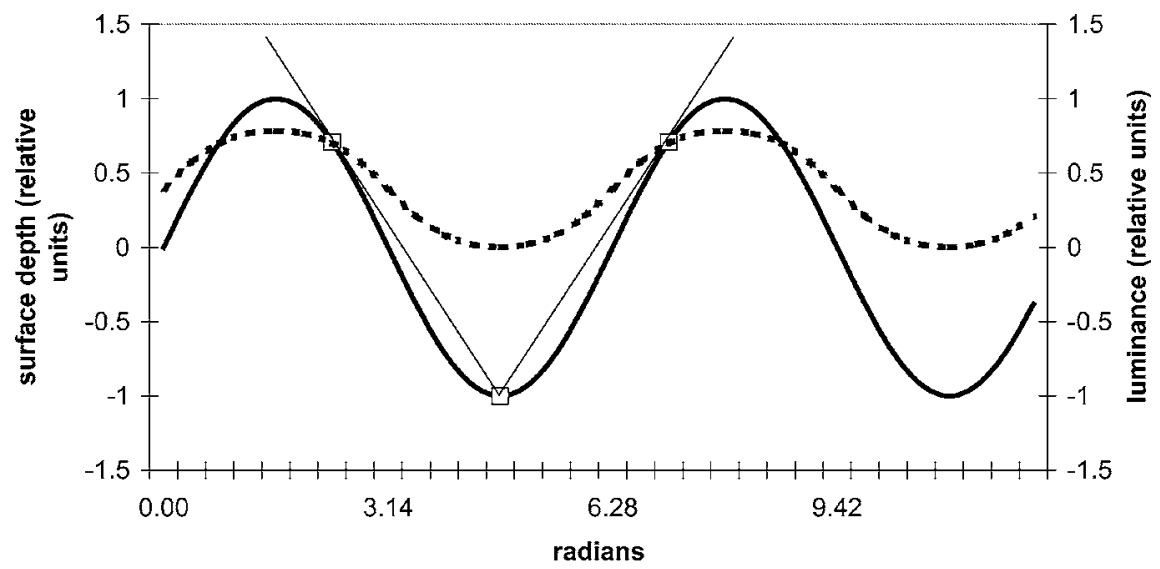

surface $\rightarrow \square-$ limit ray $=$ = - |uminance

(b)

Figure 1. (a) Theoretical distributions of image luminance for a one-dimensional sinusoidal (corrugated) surface illuminated from a single light source (ignoring mutual reflections and shadows). The thick black line shows a cross-section of the surface. The different style curves indicate the luminance profiles expected when the angle $(\alpha)$ between the incident light source and the plane of the surface (horizontal in this example) varies from $0 \mathrm{deg}$ to $\pm 67 \mathrm{deg}$. When lighting is directly from above ( $\alpha=0 \mathrm{deg}$ ), the second harmonic predominates in the luminance waveform in the image. When illumination is from the side ( $\alpha \neq 0 \mathrm{deg}$ ), alternate sides of the sinusoid are shaded, and so the first harmonic predominates in the luminance waveform in the image. (b) Theoretical distribution of image luminance for a one-dimensional sinusoidal (corrugated) surface illuminated diffusely from above. A point in the trough of the sinusoid receives light from within the 'limit rays' depicted (ignoring mutual reflections). For a point on the peak of the sinusoid, light is received from a wider angle, nominally $180 \mathrm{deg}$. Thus more light is received at the peak than the trough, resulting in a 'darker is deeper' luminance waveform in the image with a dominant first harmonic. See text for details. 


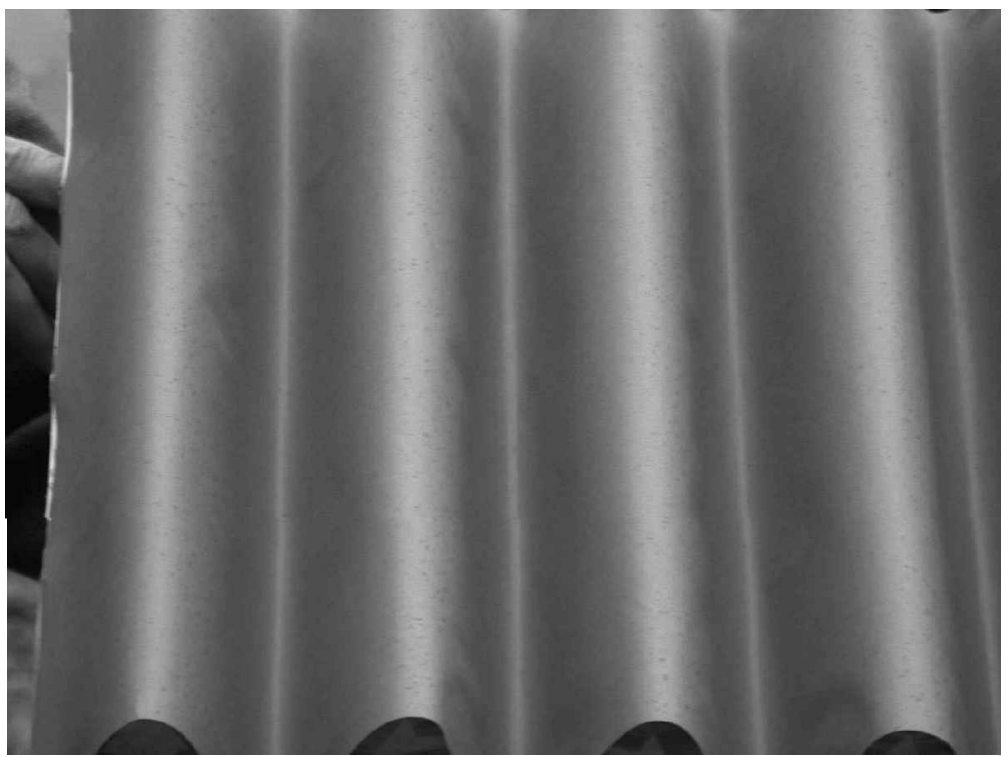

(a)

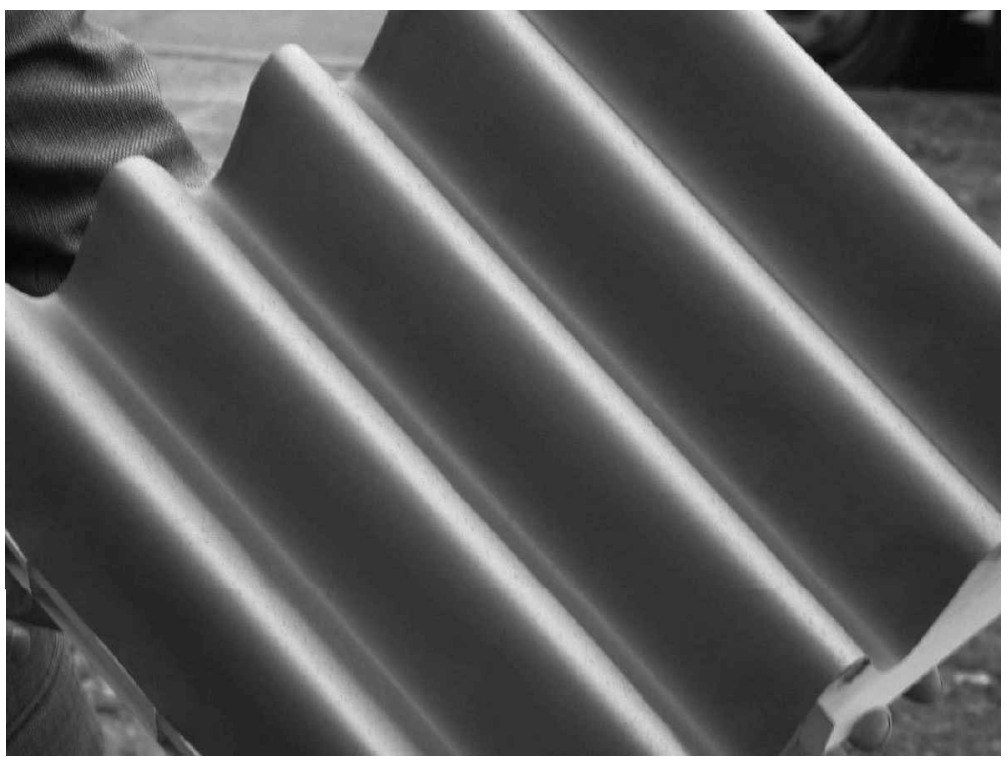

(b)

Figure 2. Digital photographs of a corrugated surface taken under a grey sky. (a) Approximate tilt $45 \mathrm{deg}$, slant 0 deg rotation 0 deg. (b) Approximate tilt $45 \mathrm{deg}$, slant 0 deg, rotation $45 \mathrm{deg}$. (c) Measured grey scale (luminance) profiles perpendicular to the corrugations for surfaces depicted in $2 \mathrm{a}$ (black) and $2 \mathrm{~b}$ (white) aligned to the surface troughs. For both views, the luminance waveform has obvious $f$ and $2 f$ components. The maximum luminance of the $f$ component corresponds to the surface peaks, and the maximum luminance of the $2 f$ component corresponds to the surface peaks and troughs. The apparent phase shift of the $f$ peak in the two waveforms is due to perspective. 


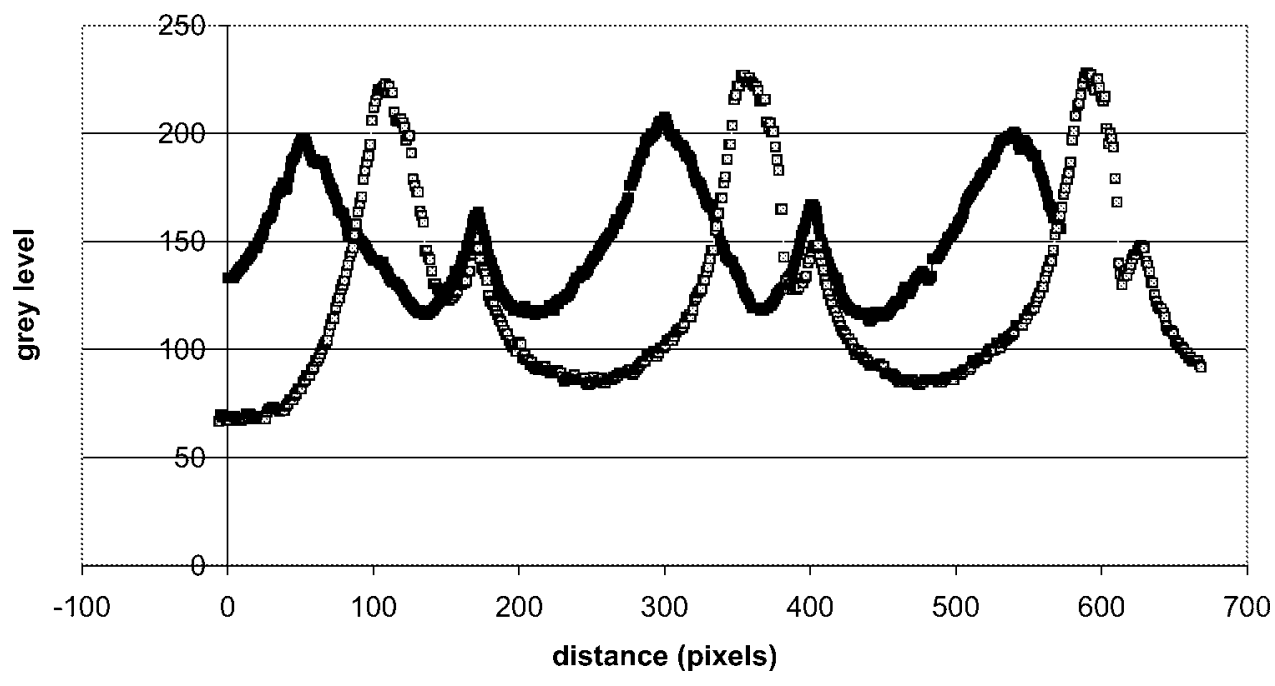

(c)

Figure 2. (Continued).

relationships to the surface (Fig. 2c). Standard matt papers are uniform but not Lambertian. The directional component in the surface reflectance function is clearly evident, and this enhances the $2 f$ component. Some phase relationships do not occur in the image. For the $f$ component, the luminance peak cannot be located in the surface trough. For the $2 f$ component, the peaks cannot be located anywhere but the peak and trough of the surface. If the peak of the surface is at phase angle $\varphi$, then the peak of the $f$ component must lie between $\varphi-\pi / 2$ and $\varphi+\pi / 2$, and the peaks of the $2 f$ component must be located at $\varphi$ and $\varphi+\pi$.

\section{METHODS}

\section{Apparatus}

Stimuli were presented on two large $X-Y$ displays (HP 1304A), each of which was viewed by one eye only, in a modified Wheatstone stereoscope configuration via front-surface mirrors (Rogers and Graham, 1982). An Apple Mackintosh IIx computer both generated and controlled the stimuli presented on the two displays with eight-bit luminance resolution. The horizontal disparity of the images presented to the observer could be manipulated electronically by means of an analogue technique in which the output of a Wavetek function generator (under computer control) was used to sinusoidally modulate the x-deflections of the two displays by equal amounts but in opposite directions. This allowed the horizontal position (and thus the horizontal disparity) of each row of pixels to be specified with sub-pixel accuracy. The viewing distance was $57 \mathrm{~cm}$ and was maintained by means 
of a chin rest. At this distance each image subtended 25 by 20 deg arc and individual pixels subtended 2.5 arc min.

\section{Stimuli}

Two-dimensional, random dot patterns were produced by assigning individual screen pixels to be 'black' or 'white' with equal probability. This resulted in a $50 \%$ density dot pattern to which a sinusoidal disparity modulation could be applied (see above) in order to produce a disparity-defined grating, the spatial structure of which was only visible when the display was viewed binocularly. The spatial frequency, phase and modulation depth (peak-to-trough disparity) of the stereoscopic waveform were under experimenter control. All disparity gratings were horizontal and their spatial frequency was either $0.2 \mathrm{c} / \mathrm{deg}$ or $0.4 \mathrm{c} / \mathrm{deg}$. A luminance grating could be combined additively with the random dot patterns and hence with the disparity grating. The spatial frequency, phase and modulation depth (contrast) of the luminance grating could be specified independently of the disparity grating. The luminance grating was always horizontal, its spatial frequency was $0.4 \mathrm{c} / \mathrm{deg}$ and its Michelson contrast was either $0 \%$ (uniform background), $5 \%$, or $50 \%$.

In the $f$ conditions, the spatial frequency of the luminance grating was always the same as that for the disparity grating (i.e. $0.4 \mathrm{c} / \mathrm{deg}$ ). The relative phase of the two gratings was either consistent with the depiction a corrugated surface from shapefrom-shading assuming oblique top lighting from a single source and a uniform reflectance Lambertian surface (C) or inconsistent with shape-from-shading (IC), that is, deeper is brighter'. In the $\mathrm{C}$ condition, adjacent peaks and troughs of the luminance grating were spatially aligned with the midpoints (zero-crossings) of the slopes of the disparity grating. In the IC condition, there was a dark bar on the peak of the disparity grating.

In the $2 f$ conditions, the spatial frequency of the luminance grating was $0.4 \mathrm{c} / \mathrm{deg}$ but that of the disparity grating was $0.2 \mathrm{c} / \mathrm{deg}$ (i.e. a ratio of $2: 1$ ). The relative phase of the luminance grating and the disparity grating was either consistent with perpendicular front lighting from a single source (C) or inconsistent with shapefrom-shading (IC). In the $\mathrm{C}$ condition, the luminance peaks were spatially aligned with the disparity peaks and troughs, and the luminance troughs were aligned with the disparity zero crossings. In the IC condition, the luminance peaks were coincident with the zero crossings of the disparity grating, and the luminance troughs were aligned to the peaks and troughs of the disparity grating. Luminance variations of the IC type would tend to indicate variations in surface reflectance rather than shading.

\section{Psychophysical procedure}

A 2-Alternative-Forced-Choice (2AFC) procedure was used. Each trial consisted of two intervals of 0.5 seconds duration, each of which contained both a random dot pattern and a luminance grating. In one of the two intervals, chosen at random, a 
disparity grating was also present and the observer's task was to indicate which interval contained the disparity grating. The luminance grating was always the same in both intervals. Five equally spaced disparity modulation-depth values were presented randomly 10 times each in order to generate a psychometric function. The disparities were chosen on the basis of pilot trials so that responses spanned the range from chance $(50 \%)$ to perfect performance (100\%). Four separate determinations were made for each data point. The data were fitted by a Weibull (1951) function, and the mean and standard error of the peak-to-trough disparity corresponding to the $75 \%$ correct threshold level were evaluated from the fitted curves. The independent variables were the relative phase of the luminance and disparity gratings, and the contrast of the luminance grating. The dependent variable was the threshold for detection of the disparity grating.

\section{Procedure: qualitative observations}

For qualitative judgements, the stimuli were printed from the computer display onto white paper using a laser printer (300 dpi) and presented in an Asher Law stereoscope consisting of two positive lenses, corrected to a viewing distance of $30 \mathrm{~cm}$, providing fusion under uncrossed disparity viewing. Each laser dot subtended 0.95 arc min, and the resolution of individual laser dots was below threshold in the optical arrangement used. Two stereoscopic pairs were presented simultaneously, one above and one below, so observers could compare the upper with the lower fused stereogram. The modulation of the luminance grating was $50 \%$. The disparity grating was well above threshold and appeared steep-sided. Unlike the analogue display it has no sub-pixel disparities: thus the disparity waveform is a stepped approximation to a sinusoid. One of the stereograms (upper or lower, selected at random) had disparity and shading (luminance) information that were both consistent with the depiction of a corrugated surface (C). For the other stereogram these two sources of information were inconsistent (IC).

It was confirmed that the observers saw the stimuli as three-dimensional. Naïve observers were asked the following questions. (1) Do these stimuli look threedimensional, that is, having depth? (2) Do the stimuli look like corrugated surfaces? Only observers that responded 'yes' to both questions were included. They were then asked a realism question and an apparent depth question (order randomised). The realism question was: Which surface, upper or lower, looks more like a real surface made out of corrugated paper or cardboard? The apparent depth question was: Which surface has the greater difference in depth between the peaks and troughs of the corrugations? Every observer made judgements about the $f$ condition (Fig. 3) and the $2 f$ condition (Fig. 4).

\section{Observers}

The two authors (MW, TL) acted as psychophysical observers. Both had normal or corrected-to-normal vision. For qualitative observations, an opportunity sample of 

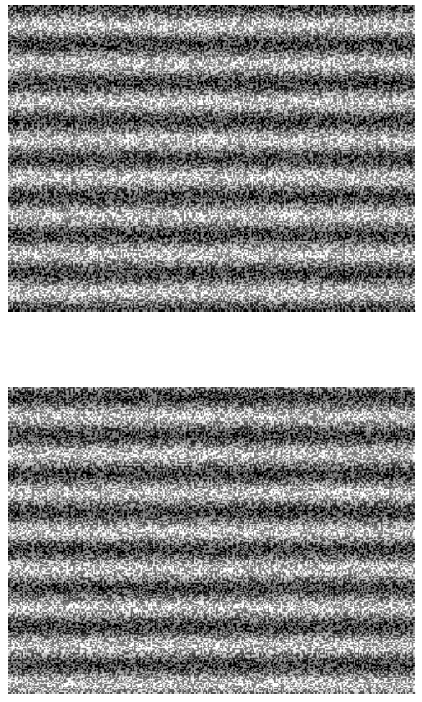

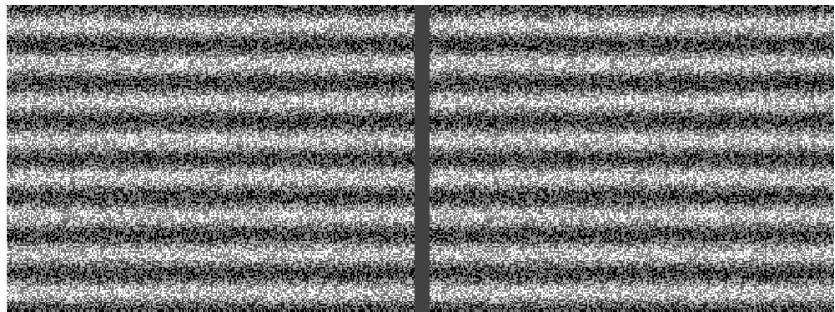

(a)

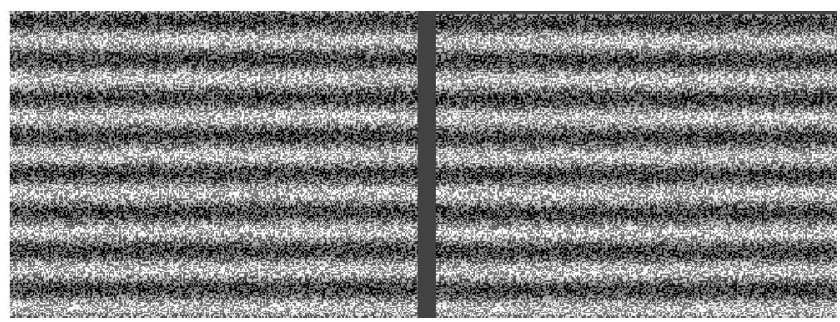

(b)

Figure 3. The triple stereoscopic images are displayed so that the left hand pair of the three images is correct under uncrossed fusion and the right hand pair is correct under crossed fusion. Each pair of fused stereoscopic half-images depicts a suprathreshold disparity grating with a superimposed luminance grating of the same spatial frequency. In (a) the shading pattern approximates to that consistent with a sinusoidal corrugated surface illuminated from above by a single oblique source. In (b) the pattern of shading is inconsistent with illumination of a uniform reflectance corrugated surface by a directional or diffuse light source. This description refers to fusion in a stereoscope or with divergent free fusion. With convergent free fusion the disparity grating (and apparent direction of the light source) is reversed.

25 naive observers was used, consisting of University students and staff (age 19-48: 17 female, 8 male). All had normal acuity. Those unable to see depth in random dot stereograms were excluded.

\section{RESULTS}

Experiment 1. Stereoscopic thresholds for detection of a disparity grating in the presence of texture lines

As set out in the Introduction, we wished to consider in a broad sense whether monocular periodic stimuli interact with disparity gratings. A variety of cues, unrelated to depth, might also show phase-specific interactions with disparity, for example through spatial cueing of locations of the maximum and minimum disparities. To test this hypothesis a stimulus was devised that marked the locations of the peaks and troughs of the disparity grating, but contained no shading information.

This stimulus was composed of a set of extended horizontal lines each of which differed from the remainder of the display only in terms of the granularity of the 

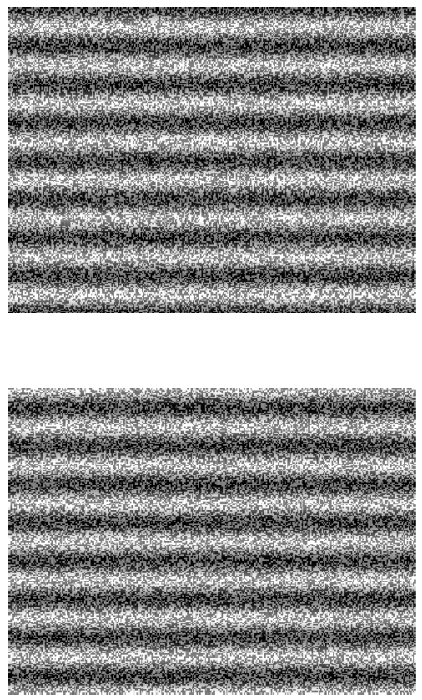

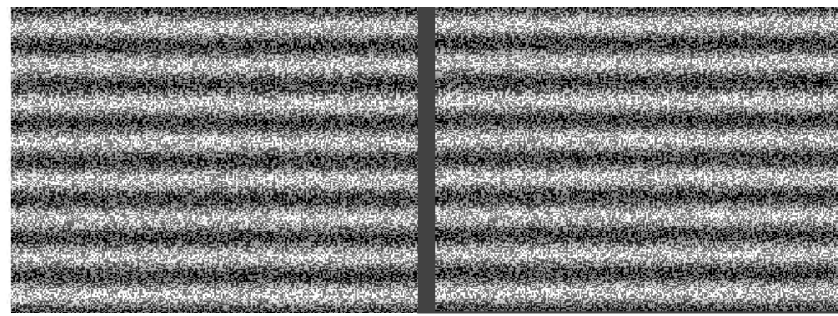

(a)

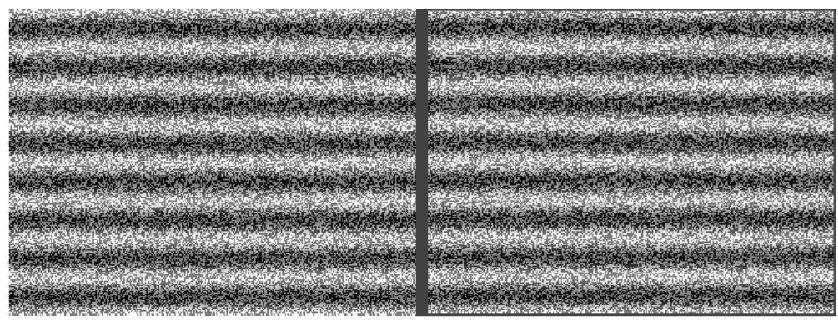

(b)

Figure 4. The triple stereoscopic images are displayed so that the left hand pair of the three images is correct under uncrossed fusion and the right hand pair is correct under crossed fusion. Each pair of fused stereoscopic half-images depicts a suprathreshold disparity grating with a superimposed luminance grating of the same spatial frequency. In (a) the shading pattern approximates to that consistent with a sinusoidal corrugated surface illuminated by a single perpendicular source. In (b) the pattern of shading is inconsistent with directional or diffuse illumination of a corrugated surface of uniform reflectance.

random-dot texture of which it was composed. This was achieved by increasing the widths, but not the heights, of consecutive noise dots within a row to 4 pixels (10 arc min) to create a line of pixels that was distinguished by texture rather than luminance. The texture within the line was still random, but each 'dot' was elongated to a row of four pixels. These horizontal texture-defined lines of one pixel height were clearly visible to the observers. They were spaced periodically within the display to mark explicitly the successive vertical positions of either the zerocrossings or peaks and troughs of a horizontal disparity grating. Data were obtained for two spatial frequencies of the disparity grating, $0.2 \mathrm{c} / \mathrm{deg}$ and $0.4 \mathrm{c} / \mathrm{deg}$. The spatial frequency of the texture grating was $0.4 \mathrm{c} / \mathrm{deg}$.

The results for two observers are shown in Fig. 5. Both observers showed superior performance when the texture lines marked the troughs of the disparity grating (in the $f$ condition), or the peaks and troughs (in the $2 f$ condition).

The significance of the differences in mean thresholds apparent in Fig. 5 was tested by analysis of variance. Factors in the ANOVA were: subjects (MW, TL) $\times$ disparity spatial frequencies $(0.4,0.2 \mathrm{c} / \mathrm{deg}) \times$ phases (peak/trough, zero crossing, control). Main effects and two-way interactions were analysed. It was found that the two observers differed overall in their stereoscopic thresholds $(F=14.6$; $d f=1,5 ; p<0.05)$, and that stereoscopic thresholds differed also for $0.4 \mathrm{c} / \mathrm{deg}$ 


\section{subject MW}

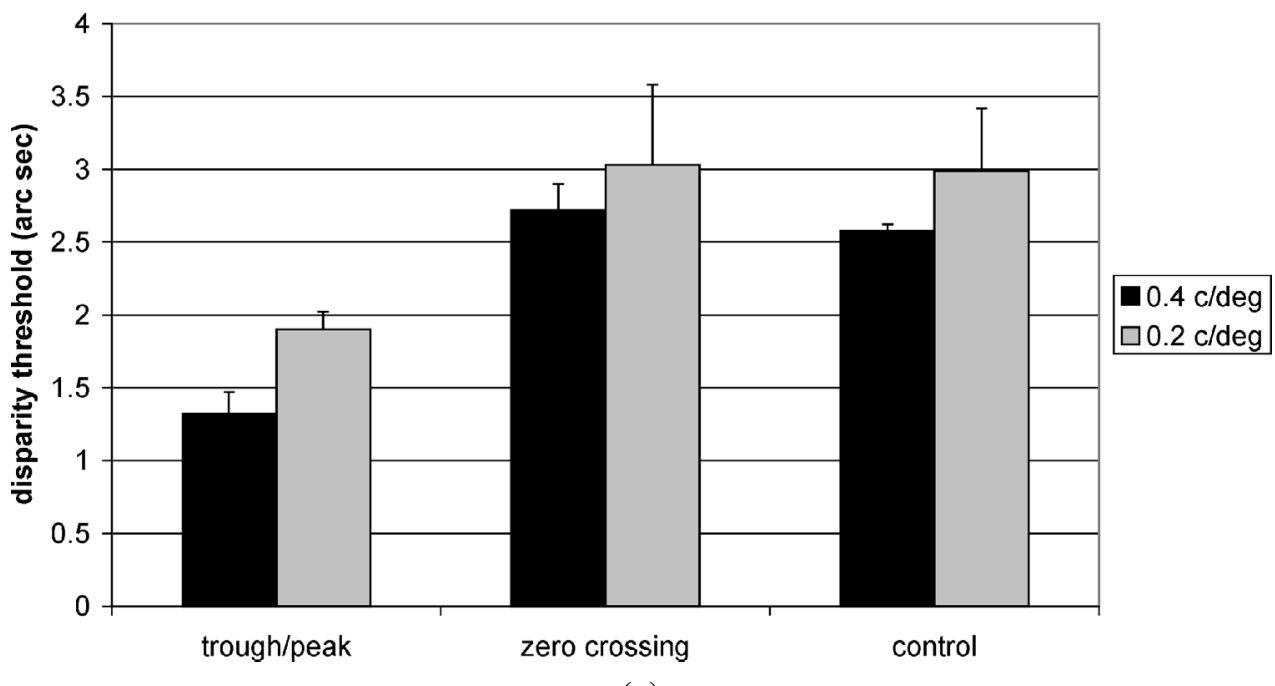

(a)

\section{subject TL}

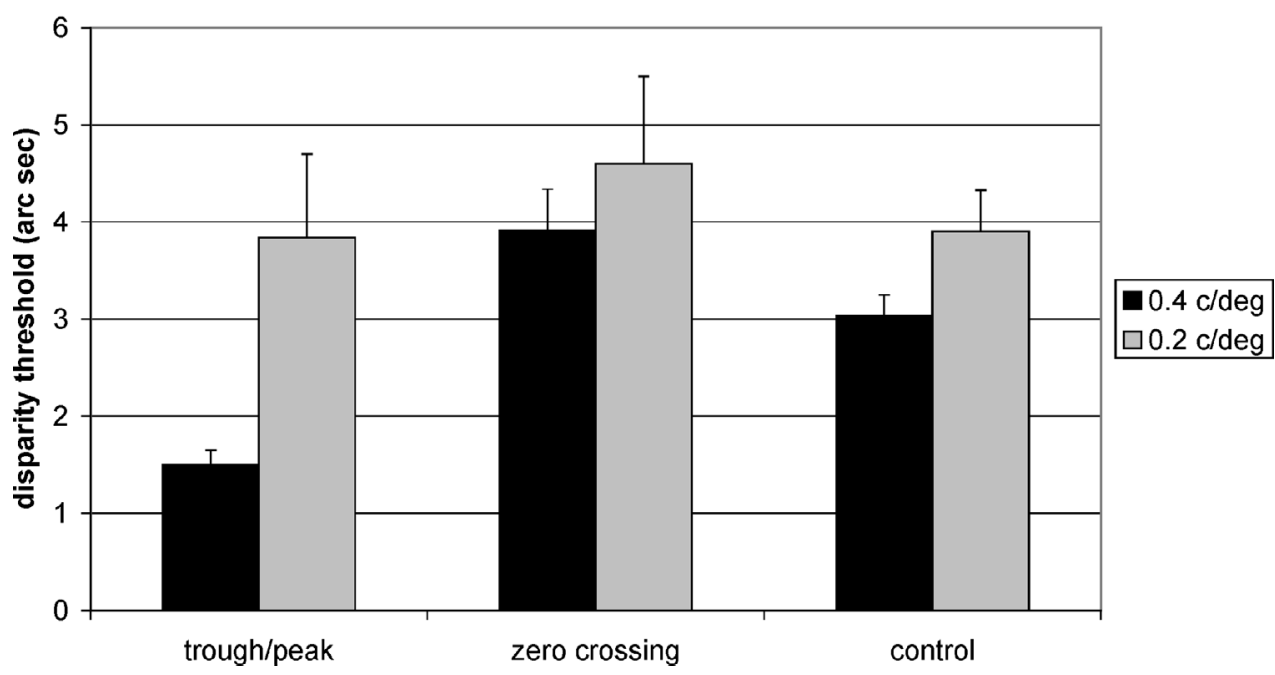

(b)

Figure 5. Stereoscopic thresholds in arc sec for the detection of a disparity grating in the presence of explicit spatial cues or markers (extended horizontal texture-defined lines). Results are plotted separately for observers MW (a) and TL (b). When the disparity spatial frequency was $0.4 \mathrm{c} / \mathrm{deg}$ (black bars), the texture lines were located either on the troughs of the disparity grating (left) or the zero-crossings (middle). The texture-defined lines were absent in the control condition (right). When the disparity spatial frequency was $0.2 \mathrm{c} / \mathrm{deg}$ (grey bars), the texture lines marked either the peaks and troughs (left), the zero-crossings (middle) or were absent (right). The vertical lines above each bar represent +1 SEM. 
and $0.2 \mathrm{c} / \mathrm{deg}$ gratings $(F=10.1, d f=1,5 ; p<0.05)$. The key comparison for the experimental hypotheses is the main effect of phases which was significant $(F=9.6, d f=1,5 ; p<0.05)$. The interactions of subjects with phases and frequencies were not significant. Post-hoc comparisons showed that the trough/peak condition had lower thresholds than the zero crossing condition (mean difference $=$ 1.4 arc sec, $p<0.05$, Scheffé test).

The texture lines influenced the appearance of the display. In the $f$ condition, the texture lines in the troughs segmented the disparity grating into parallel strips. The apparent depth of the textured lines could be compared with the midpoints of the adjacent intervening strips. In the $f$ control condition, the texture line marked alternate zero crossings, and thus gave no direct cue to the location of the disparity peaks and troughs. In the $2 f$ condition, the texture lines divided the disparity grating into half-cycles, and here the peak/trough condition was superior to control, as the disparity of each line could be compared with its neighbouring lines. In the control condition, the texture lines did not directly indicate the extremes of the disparity grating.

Experiment 2. Stereoscopic thresholds for detection of a disparity grating in the presence of a superimposed luminance grating

Disparity thresholds were measured for disparity gratings of 0.2 and $0.4 \mathrm{c} / \mathrm{deg}$, with added luminance gratings of $0.4 \mathrm{c} / \mathrm{deg}$ under two experimental conditions of relative phase ( $\mathrm{C}$ and IC), and at two luminance contrast levels (5\% and 50\%). There were also four control conditions $(0.2 \mathrm{or} 0.4 \mathrm{c} / \mathrm{deg}, 0 \%$ luminance contrast, or $50 \%$ luminance contrast with random phase).

Stereoscopic thresholds were generally somewhat lower for some of the conditions with an added luminance grating in specific phase than for control conditions. There was no evidence of masking of the disparity grating by the luminance grating.

Because of some variability across conditions, ANOVA was conducted on all the data from Experiment 2 to see which of the differences between conditions (if any) were consistent across observers. Mean data are summarised for both subjects in Fig. 6, and they are pooled across 5\% and 50\% contrast because the ANOVA (below) showed that these contrast differences were not significant. It can be seen from Fig. 6 that disparity thresholds are lower in the inconsistent conditions than the controls. The consistent conditions seem to be intermediate between inconsistent and control.

The factors in the ANOVA were: subjects (MW, TL), disparity spatial frequencies $(0.4,0.2 \mathrm{c} / \mathrm{deg})$, phases (consistent, inconsistent, control) and contrasts $(0 \%, 5 \%$, $50 \%)$. It was found that the two observers differed overall in their stereoscopic thresholds $(F=36.7 ; d f=1,12 ; p<0.0005)$, and that stereoscopic thresholds differed also for $0.4 \mathrm{c} / \mathrm{deg}$ and $0.2 \mathrm{c} / \mathrm{deg}$ disparity gratings $(F=26.2, d f=1,12$; $p<0.0005)$. The main effect of contrasts was not significant $(F=1.6, d f=2,12$; $p=0.23)$. 


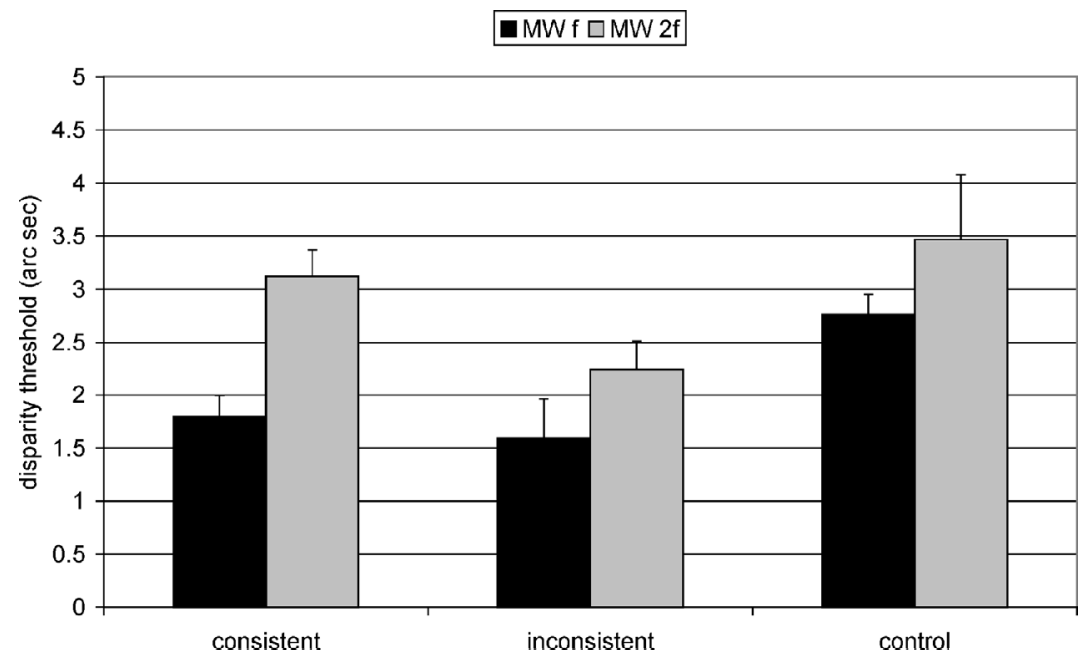

(a)

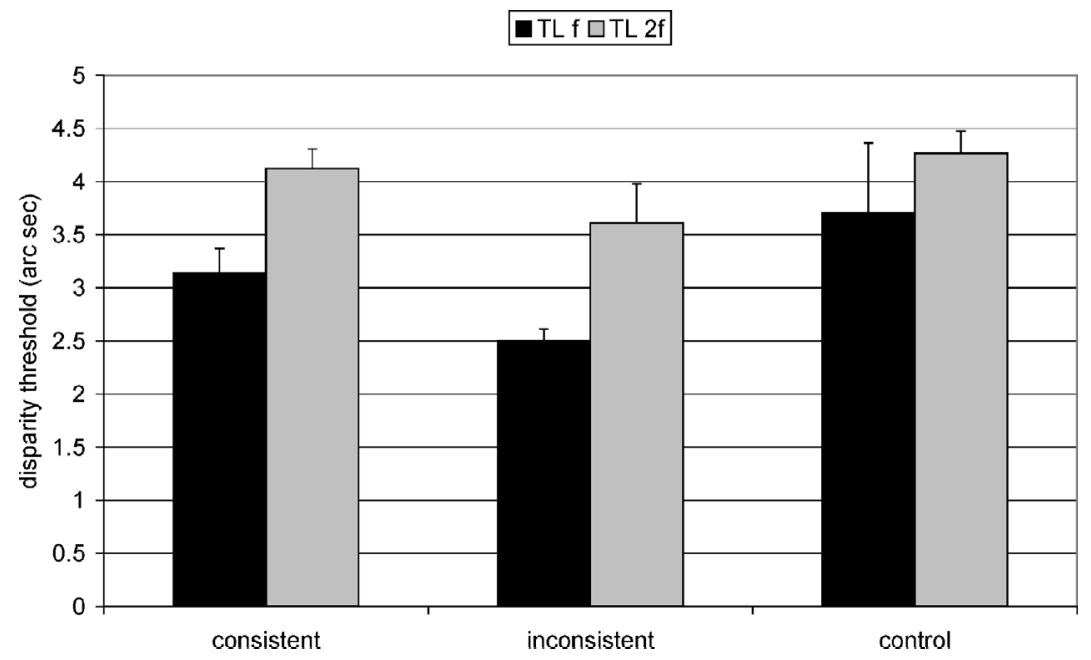

(b)

Figure 6. Stereoscopic thresholds in arc sec for the detection of a disparity grating in the presence of a luminance grating with either consistent or inconsistent patterns of shading appropriate to a surface corrugated in depth. Results are plotted separately for observers MW (a) and TL (b). For the consistent (left) and inconsistent (middle) conditions thresholds were measured in presence of a luminance grating with a contrast of either $5 \%$ or $50 \%$. As there was no statistically significant difference in performance for these two contrast levels the data were pooled and the mean thresholds are plotted in the figure. For the control conditions (right) disparity thresholds were measured either in the absence of a luminance grating (equivalent to $0 \%$ contrast) or with a luminance grating of $50 \%$ contrast and random spatial phase. Again, as there was no statistically significant difference in performance for these control conditions, the data were pooled to derive a mean threshold. The spatial frequency of the luminance grating (when present) was $0.4 \mathrm{c} / \mathrm{deg}$ and that of the disparity grating was either $0.4 \mathrm{c} / \mathrm{deg}$ ( $f$ indicated by the black bars) or $0.2 \mathrm{c} / \mathrm{deg}$ ( $2 f$ indicated by the grey bars). The vertical lines above each bar represent +1 SEM. 
The key comparison for the experimental hypotheses is that between the consistent, inconsistent and control phase conditions. The main effect of phases was significant $(F=8.4, d f=2,12 ; p=0.005)$. Post-hoc comparisons showed that the inconsistent phases had lower thresholds than the control (mean difference $=$ 1.1 arc sec, $p<0.001$, Scheffé test). Further analysis showed that there was an effect of phase on both 0.4 and 0.2 c/deg disparity gratings, as can be seen from Fig. 6. The inconsistent $f$ combination had lower thresholds than the $0.4 \mathrm{c} / \mathrm{deg}$ control ( $p=0.05$, Scheffé test), and the $2 f$ combination showed lower thresholds than the $0.2 \mathrm{c} / \mathrm{deg}$ control in both consistent $(p<0.05$, Scheffé test) and inconsistent $(p<0.05$, Scheffé test) phases.

There were no significant interactions between subjects, frequencies, phases and contrasts. Thus the effects of phase described are not contaminated by differences between the subjects, or in the response to $5 \%$ and $50 \%$ contrast gratings.

We may also analyse further the data for the control conditions. There were two control conditions: no luminance grating ( $0 \%$ contrast condition), or an added luminance grating (50\% contrast, $0.4 \mathrm{c} / \mathrm{deg}$ ) whose relative phase was randomised on every trial. Both 0.2 and $0.4 \mathrm{c} / \mathrm{deg}$ disparity gratings were used. ANOVA revealed no significant differences between the control conditions. Thus the simple presence of a luminance grating did not affect thresholds.

To conclude, there are interactions between luminance gratings and stereoscopic thresholds. Reduced thresholds were found for fixed phase relationships relative to control conditions. However, we must reject the directional hypothesis that a luminance grating consistent with shading improves detection of the disparity grating. It was the inconsistent conditions that gave the lowest mean thresholds.

\section{Experiment 3. Effects of luminance gratings of variable relative phase on stereoscopic thresholds}

The experiment was carried out for $2 f$ gratings, where the disparity grating had a spatial frequency of $0.2 \mathrm{c} / \mathrm{deg}$ and the luminance grating a spatial frequency of $0.4 \mathrm{c} / \mathrm{deg}$. The relative phase (i.e. the difference between the absolute phases) of the luminance and disparity gratings was varied between 0 and 135 deg in steps of $22.5 \mathrm{deg}$. This means that the $0 \mathrm{deg}$ condition was 'consistent' (luminance peak coincides with disparity peak and trough) and the 90 deg condition was 'inconsistent' (luminance trough coincides with disparity peak and trough). Relative phase was randomised between blocks of 50 trials. Additionally, the absolute phase of the stereoscopic grating was randomised on every trial. Thus on initial trials it was not possible to utilise the shading pattern, or the frame of the display, to identify favoured regions of the image in order to compare disparity. Spatial cueing should be reduced by the randomisation, but cue combination effects (if present) should operate from the first trial. The control condition was simply a disparity grating without an added luminance grating (i.e. its contrast was set to $0 \%$ ). The results are shown in Fig. 7. 


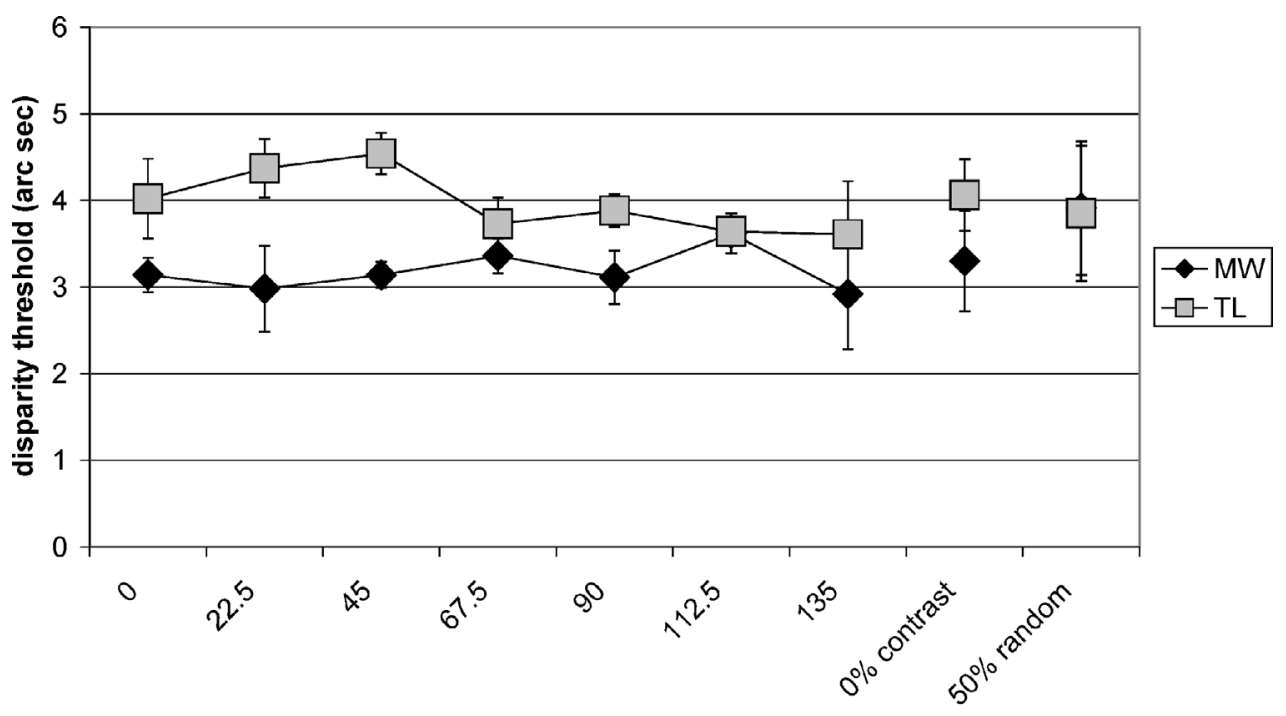

phase difference (deg)

Figure 7. Stereoscopic thresholds in arc sec for the detection of a disparity grating in the presence of a luminance grating as a function of the relative phase (difference in absolute phase) between the two gratings. Results are shown for observers MW (black symbols) and TL (grey symbols). The spatial frequency of the stereoscopic grating was 0.2 , and that of the luminance gratings was $0.4 \mathrm{c} / \mathrm{deg}$. The contrast of the luminance grating was $50 \%$, except in one control condition when it was absent (' $0 \%$ contrast'). In the other control condition (' $50 \%$ random') the spatial phase of the superimposed luminance grating was randomised on each trial. The vertical lines above and below each data point (where visible) represent \pm 1 SEM.

There were no systematic effects on stereoscopic thresholds when the relative phase angle was varied between 0 and $135 \mathrm{deg}$. In addition there was no enhancement of the stereoscopic thresholds due to the presence of a luminance grating, compared with the control condition. Comparing across Experiments 2 and 3, when we increased the positional uncertainty of the disparity grating, the improved performance of the $\mathrm{C}$ condition relative to the control was no longer evident.

\section{Experiment 4. Qualitative judgements of the appearance of combined disparity and luminance gratings}

The purpose of this experiment was to obtain some preliminary information on a substantial topic, that is, the interpretation of above-threshold combinations of shading and disparity cues. Our concern here was to test the depth interpretations ascribed to the main phase and frequency combinations of shading and disparity used in the psychophysical study, when presented in suprathreshold combinations.

In the $f$ conditions, the spatial frequency of both the luminance grating and the disparity grating was $0.4 \mathrm{c} / \mathrm{deg}$. The relative phase of the luminance grating and disparity grating was either consistent with oblique lighting (C) or inconsistent with 
shape-from-shading (IC). Stimuli used in the C and IC conditions are shown in Fig. 3.

In the $2 f$ conditions, the spatial frequency of the luminance grating $(0.4 \mathrm{c} / \mathrm{deg})$ was twice that of the disparity grating $(0.2 \mathrm{c} / \mathrm{deg})$. Again, the relative phase of the luminance grating and the disparity grating was either consistent (C) with shapefrom-shading (assuming a single perpendicular light source) or inconsistent (IC) with shape-from-shading. In the $\mathrm{C}$ condition the luminance peaks were spatially coincident with the disparity peaks and troughs, and the luminance troughs with the disparity zero-crossings. In the IC condition, the luminance peaks were spatially aligned with the zero-crossings of the disparity grating, and the luminance troughs corresponded to the peaks and troughs of the disparity grating. The suprathreshold appearance of the stimuli used in the $\mathrm{C}$ and IC conditions is shown in Fig. 4.

Results from comparisons of the suprathreshold stimuli are shown in Fig. 8. For the $f$ stimuli, the consistent stimulus was judged to be more like a real corrugated surface than the inconsistent stimulus by significantly more observers ( 22 out of 25 : exact binomial $p=0.0002$ ). However, the inconsistent stimulus was significantly more often judged as having more apparent depth than the consistent pattern (19 out of 25: exact binomial $p=0.0146$ ). For the $2 f$ stimulus, qualitative observations indicated that the consistent stimulus was significantly more often judged as the more realistic (21 out of 25: exact binomial $p=0.0009$ ). The consistent stimulus

\section{paired comparison of consistent and inconsistent shading}

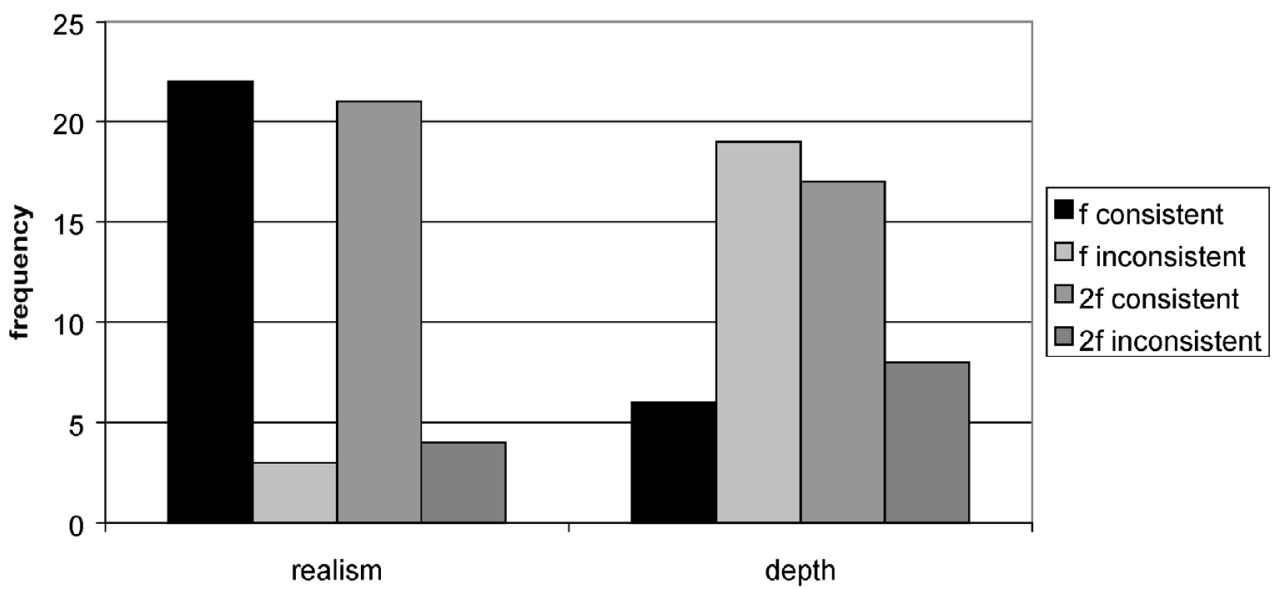

judgments of greater

Figure 8. Paired comparisons of judgements made to disparity gratings with consistent and inconsistent shading, produced by the addition of a luminance grating. The spatial frequency of the superimposed luminance grating was either the same as $(f)$ or twice $(2 f)$ that of the disparity grating. Consistent patterns were judged the more realistic by the majority of observers for both the $f$ and the $2 f$ gratings. The $f$ inconsistent gratings were, however, judged as having more depth by the majority of observers. 
was more often judged as having greater apparent depth, but not significantly so (17 out of 25: exact binomial $p=0.1078$ n.s.). The disparities in all paired comparisons were equal. Thus, the addition of a luminance grating can influence judgements of the realism and apparent depth of a surface.

\section{DISCUSSION}

\section{Interaction of luminance and disparity}

The first, and somewhat obvious, point is that the addition of a luminance grating of $50 \%$ or $5 \%$ contrast does not significantly increase thresholds for the detection of a disparity grating of the same spatial frequency. There is no masking effect, and there is a high degree of independence in the processing of luminance and disparity information. However, measurements of stereoscopic thresholds in sinusoidal disparity gratings with and without the addition of a luminance grating or textured lines showed that lowering of thresholds occurred under some conditions.

We shall now consider three possible explanations for these threshold changes: (a) interaction of shading cues with stereoscopic cues, (b) reduction of spatial uncertainty and (c) local interactions between pattern and disparity, unrelated to depth.

In Experiment 3, comparison of different relative phases showed that thresholds were independent of the relative phase of the luminance and disparity gratings of the same frequency ( $2 f$ condition) and were not enhanced relative to the control. This result is therefore a negative one for the depth-cue interaction hypothesis as well as for the local interaction hypothesis (because there is no facilitation or masking). It does not contradict the idea of spatial cueing, because the position of the disparity grating was made hard to predict, that is, there was relatively high spatial uncertainty that was uniform across conditions.

However, in Experiment 2, some phase-dependent facilitation was found. Statistically significant enhancement relative to a control condition with no luminance grating was found with an added grating of twice the spatial frequency of the disparity grating ( $2 f$ condition). However, this occurred in a phase relationship that does not occur in natural illumination of a corrugated surface. The results for an added grating at frequency $f$ demonstrated a trend in the same direction, in that inconsistent shading produced enhancement relative to the control. The differences between consistent and inconsistent shading, and between consistent shading and control did not reach significance. These observations argue against interaction of depth cues as an explanation of threshold changes since the changes, when they do occur, are not related to valid shading cues.

In a threshold task, varying the amount of signal-location uncertainty has a marked effect on the probability of a correct response (Burgess and Ghandeharian, 1984; Pelli, 1985). Where multiple depth cues are combined, the presence of suprathreshold values of one cue (e.g. shading) may reduce spatial uncertainty 
about the location of threshold values of a second cue (e.g. disparity). In this way the detection of one cue may influence the detection of a second cue without the requirement for any specific process of cue combination. In favour of this observation, it was found that, when the position of the disparity grating was not randomised (Experiment 2), more facilitation was found than when it was randomised (Experiment 3).

Other aspects of the results are more difficult to explain in terms of positional uncertainty effects. In terms of relative phase, the phase uncertainty of the disparity grating is reduced by the same amount by a luminance grating whatever its relative phase, so long as the relative phase is consistent within a block of trials. However, the determination of position is not based upon phase, but on luminance features. Georgeson and Freeman (1997) showed that in a compound grating, location of features was close to but not exactly aligned with the zero-crossings, peaks and troughs, and was well predicted by a set of even- and odd-symmetric filters. There are presumably features in the luminance grating that could reduce uncertainty about the location of the disparity peaks and troughs, though there is no reason a priori to predict that these effects would be greater for IC rather than $\mathrm{C}$ patterns. We further examined the data to look for more complex patterns of facilitation and masking.

The balance between facilitatory and masking interactions in luminance gratings is known to be contrast-dependent. Legge and Foley (1980) studied contrast thresholds for a standard sine wave grating in the presence of masking gratings of a wide range of spatial frequencies and contrasts. For high contrast masks at all spatial frequencies, thresholds increased according to a power function of masking contrast. For low contrast masks of similar spatial frequency, thresholds were reduced. This is the well-known 'dipper function', and it may be utilised to study whether visual mechanisms are independent. For example, Schofield and Georgeson (1999) studied sensitivity to luminance modulated (LM) gratings (first order) and contrast modulated (CM) gratings (second order) in the presence of white noise. They found facilitation of LM by LM and of CM by CM, but not of CM by LM or of LM by CM. This was strong evidence of independent mechanisms for the detection of first-order and second-order gratings. Applying the same argument to the present study, a luminance grating of $50 \%$ is strongly above threshold, so masking rather than facilitation of the disparity grating would be expected. From other measurements on detection of gratings in noise, and the data of Schofield and Georgeson (1999), we estimate our thresholds for the detection of a $0.4 \mathrm{c} / \mathrm{deg}$ stationary luminance grating in $50 \%$ noise to be between $1 \%$ and $2.5 \%$. At $5 \%$ contrast, the luminance grating (with $50 \%$ noise modulation) was therefore estimated to be $2-5$ times threshold contrast. Thus it is possible that facilitation rather than masking should be the outcome at $5 \%$ if the luminance and disparity mechanisms interact. The results of Experiment 2 showed no statistically significant effect of contrast although the differences in mean thresholds show a trend that suggests facilitation at low contrast. With $5 \%$ contrast gratings the threshold (mean 
of 2 observers and 2 phases) for the $f$ condition was 1.9 arc sec, compared with 2.6 arc sec at $50 \%$ contrast and 3.3 arc sec at $0 \%$ contrast, but these differences were not entirely consistent across observers and phases hence were non-significant. The $2 f$ stereoscopic thresholds showed no tendency for a difference between contrasts. The lack of significant masking effects at $50 \%$ contrast is clearly established by our data. The lack of facilitation at $5 \%$ is less certain, and it would require a more extensive set of measurements to conclusively establish the presence or absence of direct facilitation at near-threshold contrasts.

Also, in Experiment 1, texture-defined lines marking the positions of adjacent peaks and troughs of the disparity grating were found to reduce thresholds. Here, facilitation occurred when the line stimuli coincided with the peaks and/or troughs of the disparity grating. Consider that in order to achieve minimum thresholds, the observer must locate (and compare) the peaks and troughs of the disparity grating. Maxima and/or minima of disparity in a sinusoidal waveform may be more accurately determined when they are explicitly marked (peak/trough condition), rather than when their position is implicitly specified relative to a luminance or texture feature (zero-crossing condition). Also, the lines appear to segment the display, and it is known that segmentation into parts is more effective at minima of local curvature than at zero crossings (Hoffman and Richards, 1984). Moreover, it has been known for many years that maxima of curvature in a three-dimensional shape are important in defining its general shape (Attneave, 1954), and recently Bertamini (2001) has shown that position judgements are most accurate for maxima of curvature. Kingdom et al. (2001) showed that segmentation of stereoscopic surfaces was facilitated by spatial frequency differences. Our experiments indicate that texture lines may influence the threshold through segmentation of regions or locations for comparison.

It was satisfactorily shown that there was no facilitation or masking from the mere presence of a luminance grating (Experiment 2). When the relative phase was randomised on every trial, there was no superiority (or inferiority) of the combined stimulus over the disparity grating alone. With stable relative phase, more facilitation occurs with specific phase relationships. This seems to imply that for luminance gratings also, the segmentation hypothesis gives a possible explanation, and that luminance patterns influence the way that disparity information is pooled and compared across space.

Finally, in Experiment 4, a luminance grating was perceptually fused with a disparity grating to form a unitary representation of a corrugated, shaded surface when both gratings were above threshold. Moreover, relative phase influenced subjective judgements of realism and apparent depth of the stereoscopic surface: realism may be difficult to define, but it was a judgement that naïve observers could readily carry out in a $2 \mathrm{AFC}$ task. The results imply that a luminance grating whose spatial phase is consistent with single-source illumination of a corrugated surface gives a clearer or more cogent surface percept than a luminance grating with an inconsistent phase. In other words, participants seemed to have some 
implicit knowledge of the possible shading appearances of a real surface. A more extensive study might determine the conditions under which a luminance variation is interpreted as shading rather than variation in surface reflectance.

The majority of naïve observers judged inconsistent combinations of $f$ stimuli as having greater apparent depth. This was not expected if the depth cues from shading and disparity combine in some linear fashion, but it is consistent with the segmentation argument developed above. A high-contrast grating segments perceptually as alternate light and dark bars. The broad peaks and troughs are localisable features, and when they coincide with the peaks and troughs of the disparity grating, the grating bars differ in depth. When they are combined with the zero-crossings of the disparity grating in the control condition, the most prominent luminance features do not differ in average depth.

To conclude, the results do not support the idea of depth-cue combination. They are consistent with the idea of spatial cueing reducing positional uncertainty for optimal points on the disparity waveform, but the presence of some phase-specific effects also suggests local interactions between luminance or texture patterns and the processing of disparity.

\section{Interactions between shading and disparity: methodological considerations}

Subthreshold summation may provide a more sensitive test of cue combination (specific to spatial phase) or local facilitation (unspecific to spatial phase) since the cueing or segmentation effect of a suprathreshold pattern would be eliminated.

For combinations of disparity gratings, Schumer and Ganz (1979) showed that gratings of different spatial frequency behaved as independent signals, such that a compound grating was detected when either of its components reached an independent threshold. From the results reported above, the most likely outcome is that the luminance and disparity information would serve as independent signal sources and the detection of the combined signal would be superior to separate detection, through the principles of signal detection theory (Green and Swets, 1966). Where different visual components stimulate different visual mechanisms, the psychophysical performance is usually consistent with probability summation. For example, Meese and Williams (2000) have shown that psychophysical performance for the detection of compound stimuli that could vary in spatial frequency, orientation and position is consistent with probability summation models. An alternative possibility is that there is a more direct (physiological) summation. If both disparity and shading contribute to a common depth or shape mechanism, a linear combination might be expected. The properties of physiological summation could be compared quantitatively with the predictions of a probability summation model. Although there is no evidence to date of a masking effect, with a more extensive exploration of interactions close to threshold, the presence or absence of a 'dipper function' (Legge and Foley, 1980; Schofield and Georgeson, 1999) could be established across an extended range of contrasts and spatial frequencies. 
There is a modifying effect of contour on the combination of shading and disparity cues. For a sinusoidal luminance grating in a circular or rectangular aperture, local contour (the edge of the grating) usually specifies flatness. A sinusoidal grating does not usually look like a sinusoidal surface. It may be made to appear threedimensional by the addition of a sinusoidal contour of the right frequency and phase (providing a local orientation prior), or, as shown here, by the addition of a disparity grating. A sinusoidal grating with added noise and zero disparity looks somewhat three-dimensional, particularly in a large field, or where the straight edges of the display are not prominent. It might be argued that the luminance grating in our experiment does not systematically influence depth thresholds because it is contributing ambiguous depth information. The combination of contour and shading can give strong depth information, but in our experiment the bounding contour of the display suggested a flat display.

Combination of shading and stereo has been found in other studies. Doorschot $e t$ al. (2001) asked observers to perform surface attitude settings at multiple points in stereo photographs of real objects. From these settings depth maps were calculated. The location of the light source and the stereo base were both varied over three positions, giving nine combinations of stereo and shading information. Principal components analysis showed that the effect of shading and disparity combined in an almost linear relationship. An important theoretical issue is what kind of representation cues could be combined in, that is, whether or not different cues interact through a common representation, and what properties are encoded in such representations. For example, the visual system could, in principle, compute either a depth map containing local depth values, a map of local orientations, a map of local curvatures, or some other kind of shape representation. Recent evidence shows that human observers do not directly compute local depth from surfaces specified by disparity, shading and texture (Johnston and Passmore, 1994a, 1994b; Norman and Todd, 1996), but instead, shape may be represented in terms of higher-order properties such as local curvature. A further question then arises in cue-combination studies of whether or not different depth cues give rise to the same or different shape descriptors. There is a 3D curvature contrast effect, in which a surface appears more convex or concave as a result of spatially adjacent inducing stimuli. Using motiondefined shape, te Pas and Kappers (2001) found spatial contrast effects when the inducers contained second-order (curvature) information, or first-order (slant and tilt) information, but not zeroth-order (depth). Curran and Johnston (1996) showed similar effects for shading plus texture. They suggest that the curvature contrast effect is shape-defined.

Tittle et al. (1997) used a cue-conflict paradigm to assess the relative influence of disparity, shading and texture on judgements of curvature and shape index in quartic surfaces. They found that although binocular disparity made the strongest contribution overall, shading contributed relatively more to judgements of curvature than to judgements of shape index. Unlike te Pas and Kappers (2001) they concluded that there is no single representation of shape that mediates cue combination. 
Disparity can deliver information about depth (zero-order), about surface slant and tilt (first order) and about curvature (second order), and there is some evidence that the zero-order information is represented most accurately, giving the smallest Weber fractions (Lunn and Morgan, 1997). Shading, on the other hand, requires priors in order to be interpreted as shape. The priors include the location of the light source (Mammassian and Goucher, 2001) and the local orientation of a surface as specified by contour (Mammassian and Landy, 2001). Given those priors, it may convey zero-order (e.g. 'darker is deeper'), first-order (e.g. comparison of uniform regions) or second-order (e.g. luminance gradient) information. Other properties may be more directly equivalent for stereo and luminance patterns, such as orientation of contours, so that that illusory contours may be completed cross-modally (Poom, 2001). Thus studies seeking cue combination effects need to take into account the nature of the information that each cue contributes independently, as well as the spatial cueing and local interactions considered in this paper.

\section{CONCLUSIONS}

A luminance shading pattern may contribute to the subjective impression of the realism and solidity of a stereoscopic surface if combined in a phase relationship consistent with shape-from-shading. Furthermore, when a luminance grating and a disparity grating are spatially superimposed, the relative phase may influence judgements of subjective depth. The presence (or absence) of a luminance grating has only a small effect on the threshold for detecting the presence of a disparity modulation. At threshold, stereoscopic cues appear to override shading cues. Even when the shading pattern was at high contrast, the detection of the disparity grating was unimpaired. This is consistent with ecological optics. The role of stereoscopic information may be to convey precise shape information independent of variations in surface reflectance or shadows, thus it is particularly effective in breaking camouflage (Julesz, 1971) or providing shape information under complex illumination conditions such as those found under tree cover.

\section{Acknowledgements}

These experiments were conducted in Brian Rogers' laboratory in the Department of Experimental Psychology, Oxford University. We thank him for his support and encouragement, and for the use of his facilities.

\section{REFERENCES}

Attneave, F. (1954). Some informational aspects of visual perception, Psychological Review 61, 183193.

Bertamini, M. (2001). The importance of being convex: An advantage for convexity when judging position, Perception 30, 1295-1310. 
Bradshaw, M. F. and Rogers, B. J. (1996). The interaction of binocular disparity and motion parallax in the computation of depth, Vision Research 36, 3457-3468.

Bradshaw, M., Rogers, B. J. and De Bruyn, B. (1995). Perceptual latency and complex random-dot stereograms, Perception 24, 749-759.

Bülthoff, H. H. and Mallot, H. A. (1988). Integration of depth modules: stereo and shading, J. Opt. Soc. Amer. A 5, 1749-1757.

Bülthoff, H. H. and Mallot, H. A. (1990). Integration of stereo, shading and texture, in: AI and the Eye, Blake, A and Troscianko, T. (Eds). John Wiley.

Burgess, A. E. and Ghandeharian, H. (1984). Visual signal detection: II. Signal-locationidentification, J. Opt. Soc. Amer. A 1. 906-910.

Campbell, F. W. and Robson, J. G. (1968). Applications of Fourier theory to the visibility of gratings, J. Physiol. 197, 551-566.

Cumming, B. G., Johnston, E. B. and Parker, A. J. (1993). Effects of different texture cues on curved surfaces viewed stereoscopically, Vision Research 33, 827-838.

Curran, W. and Johnston, A. (1996). Three-dimensional curvature contrast — geometric or brightness illusion? Vision Research 36, 3641-3653.

Doorschot, P. C. A., Kappers, A. M. L. and Koenderink, J. J. (2001). The combined influence of binocular disparity and shading on pictorial shape, Perception and Psychophysics 63, 1038-1047.

Georgeson, M. A. and Freeman, T. C. A. (1997). Perceived location of bars and edges in onedimensional images: Computational models and human vision, Vision Research 37, 127-142.

Graham, M. and Rogers, B. (1982). Simultaneous and successive contrast effects in the perception of depth from motion-parallax and stereoscopic information, Perception 11, 247-262.

Green, D. A. and Swets, J. A. (1966). Signal Detection Theory and Psychophysics. John Wiley, New York.

Hoffman, D. D. and Richards, W. A. (1984). Parts of recognition, Cognition 18, 65-96.

Johnston, A. and Passmore, P. J. (1994a). Shape from shading; I. Surface curvature and orientation, Perception 23, 169-189.

Johnston, A. and Passmore, P. J. (1994b). Independent encoding of surface orientation and surface curvature, Vision Research 34, 3005-3012.

Johnston, E. B., Cumming, B. G. and Parker, A. J. (1993). Integration of depth modules: stereopsis and texture, Vision Research 33, 813-826.

Julesz, B. (1971). Foundations of Cyclopean Perception. University of Chicago Press, Chicago, IL.

Kelly, F. and Grossberg, S. (2000). Neural dynamics of 3-D surface perception: Figure-ground separation and lightness perception, Perception and Psychophysics 23, 1596-1618.

Kingdom, F. A. A., Ziegler, L. R. and Hess, R. F. (2001). Luminance spatial scale facilitates stereoscopic depth segmentation, J. Opt. Soc. Amer. A 18, 993-1002.

Langer, M. S. and Bülthoff, H. H. (2000). Depth discrimination from shading under diffuse lighting, Perception 29, 649-660.

Legge, G. E. and Foley, J. M. (1980). Contrast masking in human vision, J. Opt. Soc. Amer. 70, 1458-1471.

Lunn, P. D. and Morgan, M. J. (1997). Discrimination of the spatial derivatives of horizontal binocular disparity, J. Opt. Soc. Amer. A 14, 360-371.

Mammassian, P. and Goutcher, R. (2001). Prior knowledge on the illumination position, Cognition 81, B1-B9.

Mammassian, P. and Landy, M. S. (2001). Interaction of visual prior constraints, Vision Research 41, 2653-2668.

Marr, D. (1982). Vision, p. 244. W. H. Freeman, San Francisco, CA.

Meese, T. S. and Williams, C. B. (2000). Probability summation for multiple patches of luminance modulation, Vision Research 40, 2101-2113.

Neumann, L. and Neumann, A. (1995). Radiosity and hybrid methods, ACM Transactions on Graphics 14, 233-265. 
Norman, J. F. and Todd, J. T. (1996). The discriminability of local surface structure, Perception 25, 381-398.

Pelli, D. G. (1985). Uncertainty explains many aspects of visual contrast detection and discrimination, J. Opt. Soc. Amer. A 2, 1508-1531.

Phong, B.-T. (1975). Illumination for computer generated pictures, Communications of the ACM 18, 311-317.

Poom, L. (2001). Visual inter-attribute contour completion, Perception 30, 855-865.

Robson, J. G. (1966). Spatial and temporal contrast sensitivity functions of the visual system, J. Opt. Soc. Amer. 56, 583-601.

Rogers, B. J. and Graham, M. E. (1979). Motion parallax as an independent cue for depth perception, Perception 8, 125-134.

Rogers, B. J. and Graham, M. E. (1982). Similarities between motion parallax and stereopsis in human depth perception, Vision Research 22, 261-270.

Rohaly, A. M. and Wilson, H. R. (1999). The effects of contrast on perceived depth and depth discrimination, Vision Research 39, 9-18.

Schofield, A. J. and Georgeson, M. A. (1999). Sensitivity to modulations of luminance and contrast in visual white noise: separate mechanisms with similar behaviour, Vision Research 39, 2697-2716.

Schumer, R. and Ganz, L. (1979). Independent stereoscopic channels for different extents of spatial pooling, Vision Research 19, 1303-1314.

Te Pas, S. F. and Kappers, A. M. L. (2001). First-order structure induces the 3D curvature contrast effect, Vision Research 41, 3829-3835.

Tittle, J. S., Norman, J. F., Perotti, V. J. and Phillips, F. (1998). The perception of scale-dependent and scale-independent surface structure from binocular disparity, texture and shading, Perception 26, $147-166$.

Todd, J. T., Norman, J. F., Koenderink, J. J. and Kappers, A. M. L. (1997). Effects of texture, illumination and surface reflectance on stereoscopic shape perception, Perception 26, 807-822.

Tyler, C. W. (1998). Diffuse illumination as a default assumption for shape-from-shading in the absence of shadows, J. Imaging Sci. Technol. 42, 319-325.

Weibull, W. (1951). A statistical distribution function of wide applicability, J. Appl. Mech. 18, 292297. 